- Original Article

\title{
Lower Levels of Serum Adiponectin and the T Allele of rs1501299 of the ADIPOQ Gene Are Protective against Polycystic Ovarian Syndrome in Jordan
}

\author{
Mahmoud A Alfaqih*, Yousef S. Khader, Ahmed N. Al-Dwairi, Abdallah Alzoubi, Othman Al-Shboul, Amanie Hatim \\ Department of Physiology and Biochemistry, School of Medicine, Jordan University of Science and Technology, Irbid, Jordan
}

Background: Polycystic ovary syndrome (PCOS) is a common reproductive disorder. Obesity, which is linked with lower adiponectin levels, increases a woman's risk of developing PCOS; however, the association between adiponectin and PCOS is controversial. Adiponectin levels could be affected by single nucleotide polymorphisms (SNPs) in the $A D I P O Q$ gene. This study aimed to test the relationship between serum adiponectin and PCOS in Jordan and the association between the rs2241766, rs1501299, and rs266729 SNPs in the ADIPOQ gene and PCOS.

Methods: One hundred and fifty-four women with PCOS and 149 age- and body mass index-matched normally menstruating controls were recruited. Serum adiponectin levels were measured using enzyme-linked immunosorbent assay. Genotyping was performed using polymerase chain reaction-restriction fragment length polymorphism analysis.

Results: Serum adiponectin levels were significantly lower $(\mathrm{P}=0.0064)$ in PCOS women and rs1501299 (+276 G/T) genotype distributions were significantly different $(\mathrm{P}=0.01)$ between them and normally menstruating women. Multivariate analysis revealed that adiponectin levels remained significantly lower in PCOS women $(\mathrm{P}=0.001$; odds ratio [OR], 0.9; 95\% confidence interval [CI], 0.84-0.96). The GT genotype of rs1501299 increased the risk of PCOS $(\mathrm{P}<0.001$; OR, 5.46; 95\% CI, 2.42-12.33) and increased the risk of PCOS by three-fold ( $\mathrm{P}<0.001$; OR, 3.00; 95\% CI, 1.36-6.60) relative to the TT genotype. The GG genotype increased the risk of PCOS as well $(\mathrm{P}<0.001 ; 0 R, 3: 00 ; 95 \%$ CI, 1.36-6.60).

Conclusion: PCOS is associated with lower serum adiponectin levels independent of age and body mass index. The $T$ allele of the rs1501299 (+276 G/T) SNP of the ADIPOQ gene protects against PCOS.

Keywords: Polycystic Ovarian Syndrome; Single Nucleotide Polymorphism; Adiponectin; Insulin Resistance

Received: October 10, 2017, Revised: November 18, 2017, Accepted: November 28, 2017

*Corresponding Author: Mahmoud A Alfaqih https://orcid.org/0000-0002-6383-7014

Tel: +962-2-7201000, Fax: +962-2-7095123, E-mail: maalfaqih@just.edu.jo 


\section{INTRODUCTION}

Polycystic ovary syndrome (PCOS) is a metabolic disorder that affects women during their reproductive years. The disease is heterogeneous in its clinical presentation. Diagnostic features include irregular periods, increased androgen secretion and/or polycystic ovaries. ${ }^{1)}$ Women that have PCOS are usually obese and are characterized by an android pattern of fat distribution. ${ }^{2)}$ Not surprisingly, insulin resistance is a common characteristic of women that have PCOS. This might explain why women with PCOS are at a higher risk of developing type 2 diabetes mellitus (T2DM) in their lifetime. ${ }^{3)}$ Abdominal obesity is not only a feature of PCOS, it seems to be involved in the disease etiology. ${ }^{4)}$ This is supported by the fact that PCOS patients benefit from weight reduction and the accompanying increase in insulin sensitivity. ${ }^{5}$ )

Abdominal obesity is characterized by an increase in subcutaneous or visceral fat. The contribution of subcutaneous or visceral fat to the etiology of metabolic disorders is not well-determined. ${ }^{6)}$ Recent evidence indicates that visceral fat percentage is more strongly linked than subcutaneous fat with the development of insulin resistance. ${ }^{7)}$ Most importantly, visceral fat has a different metabolic profile than subcutaneous fat, including its production of several peptide hormones collectively called adipocytokines. ${ }^{8,9)}$ Indeed, the activity of these hormones modulates the sensitivity of the surrounding tissues to insulin; therefore, their levels appear to modify the risk of developing several metabolic disorders. ${ }^{10)}$

Of the many adipocytokines secreted by visceral fat cells, adiponectin is the most abundant. ${ }^{11)}$ Adiponectin reduces the risk of developing several disorders with insulin resistance as a common feature. ${ }^{12)}$ This finding is supported by several lines of evidence; adiponectin is one of the few proteins produced by adipose tissue that is negatively correlated with obesity and the development of T2DM. ${ }^{13,14)}$ Moreover, mutations that inactivate the gene that codes for adiponectin (ADIPOQ) predispose affected individuals to the development of T2DM. ${ }^{15)}$ Given the above discussion, it is not surprising that lower adiponectin levels were linked with PCOS in some but not all populations. ${ }^{16)}$

Variations in adiponectin levels could be the result of differences in adipose tissue distribution but can be also the result of genetic variations in the $A D I P O Q$ gene that affect its activity/expression. ${ }^{17)}$ A number of single nucleotide polymorphisms (SNPs) in the ADIPOQ gene are associated with lower adiponectin expression or a predisposition to several diseases characterized by insulin resistance such as obesity $^{18)}$ and T2DM. ${ }^{19)}$ Here we examined the association between serum adiponectin levels and PCOS in a Jordanian population. Using the same population, we also evaluated the association between three SNPs in the ADIPOQ gene (rs2241766, rs1501299, and rs266729) and PCOS.

\section{METHODS}

\section{Study Design}

This was a matched case-control study. Prior to patient recruitment, the project was approved by the appropriate institutional review boards (IRB approval no., 284/2016) affiliated with Jordan University of Science and Technology (Irbid, Jordan). Participation in the study required informed consent. Recruitment took place at the gynecology clinics of King Abdullah University Hospital (Irbid, Jordan) between September 2016 and March 2017.

\section{Subjects}

Women with PCOS were recruited from the gynecology clinics at King Abdullah University Hospital. Each was diagnosed with PCOS by at least two gynecologists according to the Rotterdam guidelines, ${ }^{1)}$ which include the presence of any two of the following three criteria: (1) oligomenorrhea and amenorrhea; (2) presence of biochemical and clinical signs of hyperandrogenism; and (3) presence of 12 or more ovarian follicles or an ovarian volume $>10 \mathrm{~cm}^{3}$. Women in the control group were recruited from the same tertiary hospital but not necessarily from the gynecology clinics. Women who met the following criteria were invited to participate in the control arm of the study: (1) regular menstrual cycle quantity and duration for at least 6 months prior to enrollment; (2) no evidence of hyperandrogenism such as hirsutism or acne development; (3) ovaries that were free of any cysts on an ultrasound examination by the study gynecologist; and (4) normal hormonal profile. Women who were affected with Cushing syndrome, syndromes of severe insulin resistance, androgen-secreting neoplasms, thyroid dysfunction, congenital adrenal hyperplasia, or hyperprolactinemia were excluded from both study arms. Normally menstruating women were recruited after women with PCOS, and the two groups were matched by body mass index (BMI) and age. The PCOS women were grouped by $\mathrm{BMI}\left(<25.0,25.0-29.9\right.$, or $\left.\geq 30.0 \mathrm{~kg} / \mathrm{m}^{2}\right)$ and age $(\leq 18,19-24,25-29$, or $\geq 30$ years). Frequency matching was then performed during the recruitment of normally menstruating women.

\section{Data Collection}

During each patient's visit to the gynecology clinic, the study was explained and written consent was requested. Patients who met the eligibility criteria described above were interviewed by the attending gynecologist and relevant information was collected by a clinical research coordinator into a structured data collection sheet. Height, weight, and age were recovered from the patients' medical records. The formula BMI=weight $(\mathrm{kg}) /\left(\right.$ height $\left.[\mathrm{m}]^{2}\right)$ was used to calculate BMI.

\section{Blood and Serum Sample Collection}

The blood withdrawal was performed after an overnight fast. Blood samples were divided into an ethylenediaminetetraacetic acid (EDTA) tube ( $5 \mathrm{~mL}$ of blood; AFCO, Amman, Jordan) and a clot activator with gel plain tube ( $10 \mathrm{~mL}$ blood; AFCO, Jordan). Blood in the EDTA tube was used for the DNA extraction. To recover the serum, blood in the plain tubes was centrifuged at $4,000 \times \mathrm{g}$ for 7 minutes and later used to determine adiponectin concentrations. 
Table 1. Location and genotyping strategy of $A D I P O Q$ SNP

\begin{tabular}{llllll}
\hline \multicolumn{1}{c}{ SNP ID } & Location and base change & \multicolumn{1}{c}{ Forward primer (5' to 3') } & \multicolumn{1}{c}{ Reverse primer (5' to 3') } & Product size (bp) & Restriction enzyme \\
\hline rs266729 & Promoter region (C/G) & 5' ACTGTGGAGATGATATCTGG '3 & 5' CATTTGACAGCTACCTTGG '3 & 412 & Hha1 \\
rs1501299 & Intron2 (G/T) & 5' TGACCAGGAACCACGACTC '3 & 5' CCATCTACACTCATCCTTGG '3 & 341 & Bsml \\
rs2241766 & Exon 2 (T/G) & 5' AGTAGACTCTGCTGAGATGG '3 & 5' ACATTCTTACCTGGATCTCC '3 & 333 & BspH1 \\
\hline
\end{tabular}

SNP, single nucleotide polymorphism.

Table 2. Participants' demographic characteristics

\begin{tabular}{lccc}
\hline \multicolumn{1}{c}{ Characteristic } & $\begin{array}{c}\text { Normally } \\
\text { menstruating } \\
\text { women }(\mathrm{n}=149)\end{array}$ & $\begin{array}{c}\text { Women with polycystic } \\
\text { ovarian syndrome } \\
(\mathrm{n}=154)\end{array}$ & P-value \\
\hline Age $(\mathrm{y})$ & $24.2 \pm 5.1$ & $23.9 \pm 5.1$ & 0.50 \\
$\leq 18$ & 12.2 & 10.7 & \\
$19-24$ & 45.8 & 47.3 & 0.96 \\
$25-29$ & 25.2 & 24.0 & \\
$\geq 30$ & 16.8 & 18.0 & \\
Body mass index $\left(\mathrm{kg} / \mathrm{m}^{2}\right)$ & $26.2 \pm 5.9$ & $26.6 \pm 5.8$ & 0.80 \\
$<25.0$ & 46.0 & 45.5 & \\
$25.0-29.9$ & 28.5 & 26.5 & 0.85 \\
$\geq 30.0$ & 25.5 & 28.0 & \\
\hline
\end{tabular}

Values are presented as mean \pm standard deviation or $\%$. P-values were calculated by Student t-test.

\section{Adiponectin Measurements}

Adiponectin concentrations were determined using an enzyme-linked immunosorbent assay kit according to the manufacturer's instructions (R\&D Systems, Minneapolis, MN, USA). Absorbance at $450 \mathrm{~nm}$ was determined on an ELx800 Microplate Reader (BioTek Instruments, Winooski, VT, USA).

\section{DNA Extraction and Genotyping}

Whole blood was used to purify genomic DNA using a QIAamp DNA Blood Mini Kit purchased from Qiagen (Hilden, Germany) according to the manufacturer's instructions. Following DNA extraction, the final DNA concentration was measured spectrophotometrically using an ND-2000 Nanodrop (Thermo Scientific, Waltham, MA, USA). Polymerase chain reaction-restriction fragment length polymorphism (PCR-RFLP) was used to genotype all three SNPs of the ADIPOQ gene. The final volume of all PCR reactions was $25 \mu \mathrm{L}$. The reaction mixture contained GoTaq Green Master Mix (Promega, Madison, WI, USA), 5 ng of DNA and $0.4 \mu \mathrm{M}$ forward and reverse primers. The sequence of the primers used for genotyping each SNP, size of the corresponding PCR amplicon, and name of the restriction enzyme used for genotyping are shown in Table 1. NEB (New England BioLabs, Ipswich, MA, USA) was the source of all restriction enzymes. The undigested PCR amplicons and the products of restriction enzyme digestion were electrophoresed on a $3 \%$ agarose gel containing ethidium bromide. Ultraviolet light was used to visualize the products. The genotyping results were confirmed by direct Sanger sequencing performed at Princess Haya Biotechnology Center.

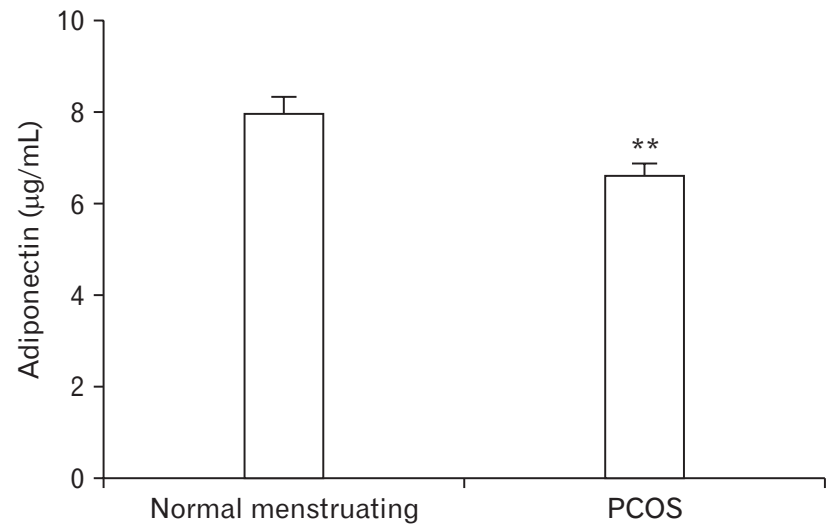

Figure 1. Adiponectin levels in normally menstruating or PCOS women. Values are presented as mean \pm standard error of the mean. Adiponectin levels were significantly lower in PCOS compared to normally menstruating women. PCOS, polycystic ovarian syndrome. ${ }^{*}$ Indicates significant differences between the two groups $(P<0.01)$.

\section{Statistical Analysis}

IBM SPSS software ver. 22.0 (IBM Corp., Armonk, NY, USA) was used to conduct the statistical analyses. Student t-test was used to identify whether significant differences existed in serum adiponectin levels between normally menstruating and PCOS women. Pearson's chi square test was used to examine whether an association existed between each ADIPOQ SNP and PCOS. Multinomial logistic regression was used in the multivariate analysis using the following variables: age, BMI, adiponectin, rs2241766, rs1501299, and rs266729.

\section{RESULTS}

\section{Demographic Data}

During the 7-month recruitment period, 154 PCOS and 149 normally menstruating women were recruited. Normally menstruating women were matched with PCOS women by BMI and age as shown in Table 2. No significant differences in BMI or age were observed between PCOS and normally menstruating women (Table 2).

\section{Association between Adiponectin, Adiponectin Gene Variations, and Polycystic Ovary Syndrome}

This study aimed to investigate whether an association exists between serum adiponectin levels and PCOS in Jordan by comparing serum adiponectin levels between normally menstruating and PCOS women. In normally menstruating women, the mean adiponectin levels were $7.916 \pm 0.3970 \mu \mathrm{g} / \mathrm{mL}$. The serum adiponectin levels in PCOS women were significantly lower $(6.582 \pm 0.2871 \mu \mathrm{g} / \mathrm{mL}, \mathrm{P}=0.0064)$ (Figure 1). 


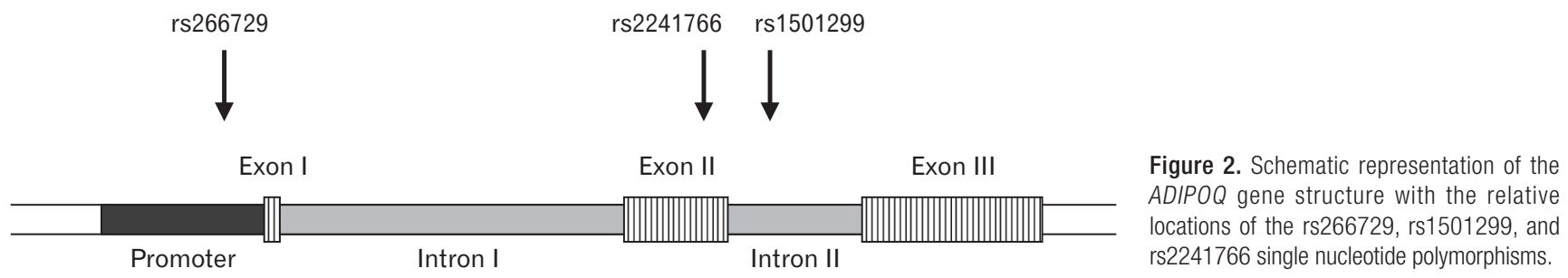

Table 3. Genotype frequencies of rs266729, rs1501299, and rs2241766 SNPs in normally menstruating and PCOS women

\begin{tabular}{cccrc}
\hline SNP ID & Genotype & $\begin{array}{c}\text { Normally menstruating } \\
\text { women }(\mathrm{n}=149)\end{array}$ & $\begin{array}{c}\text { PCOS women } \\
(\mathrm{n}=154)\end{array}$ & P-value \\
\hline rs266729 & CC & $59.1(88)$ & $54.5(84)$ & 0.37 \\
& GG & $36.2(54)$ & $38.0(58)$ & \\
& GG & $4.7(7)$ & $9.1(14)$ & \\
rs1501299 & GG & $42.3(64)$ & $40.7(62)$ & 0.01 \\
& GT & $35.6(54)$ & $49.7(77)$ & \\
& TT 2241766 & $22.2(33)$ & $9.6(15)$ & \\
& TT & $62.4(93)$ & $59.5(92)$ & 0.85 \\
& GT & $28.2(42)$ & $30.8(48)$ & \\
& GG & $9.4(14)$ & $9.1(14)$ & \\
\hline
\end{tabular}

Values are presented as \% (number). P-values were calculated by Pearson's chisquare test.

SNP, single nucleotide polymorphism; PCOS, polycystic ovarian syndrome.

The association between several SNPs of the ADIPOQ gene and insulin resistance was previously reported. ${ }^{20)}$ Considering the central role insulin resistance presumably plays in PCOS, we evaluated the association between three SNPs (rs266729, rs1501299, and rs2241766) and PCOS. These SNPs are located on different areas of the ADIPOQ gene and represent the promoter (rs266729), intron number 2 (rs1501299), and exon number 2 (rs2241766) (Figure 2). These SNPs were all genotyped using the PCR-RFLP strategy. The genotype frequencies of all three SNPs in normally menstruating and PCOS women are shown in Table 3. Our results showed significant differences in the genotype frequencies of rs1501299 between normally menstruating and $\mathrm{PCOS}$ women $(\mathrm{P}=0.01)$ (Table 3$)$.

The results above motivated us to perform a multivariate analysis with all study variables in the model. Our analysis showed that lower adiponectin levels remained associated with PCOS ( $\mathrm{P}=0.001$; odds ratio [OR], 0.9; 95\% confidence interval [CI], 0.84-0.96) (Table 4) even after the inclusion of age, BMI, rs266729, rs1501299, and rs2241766 in the model. Moreover, the above analysis also showed that the GT genotype of rs1501299 increased the risk of PCOS ( $<<0.001$; OR, 5.46; 95\% CI, 2.42-12.33) relative to the TT genotype. A similar result was observed for the GG genotype of rs1501299, where it also increased the risk of PCOS relative to the TT genotype $(\mathrm{P}<0.001$; OR, 3.00; 95\% CI, $1.36-6.60)$.

\section{DISCUSSION}

The results of this study add to a growing body of evidence that a link
Table 4. Multivariate analysis of study participants with BMI, age, adiponectin, rs266729, rs1501299, and rs2241766 as variables

\begin{tabular}{lccc}
\hline \multicolumn{1}{c}{ Variable } & Genotype & $\begin{array}{c}\text { Odds ratio } \\
(95 \% \text { confidence interval })\end{array}$ & P-value \\
\hline BMl $\left(\mathrm{kg} / \mathrm{m}^{2}\right)$ & - & $1.01(0.97-1.06)$ & 0.579 \\
Age $(\mathrm{y})$ & - & $0.96(0.92-1.01)$ & 0.132 \\
Adiponectin $(\mu \mathrm{g} / \mathrm{mL})$ & - & $0.90(0.84-0.96)$ & 0.001 \\
rs266729 & CC & 1 & \\
& GG & $2.41(0.88-6.60)$ & 0.089 \\
& GG & $1.12(0.67-1.86)$ & 0.674 \\
rs1501299 & T & 1 & \\
& GT & $5.46(2.42-12.33)$ & 0.000 \\
rs2241766 & GG & $3.00(1.36-6.60)$ & 0.006 \\
& GG & 1 & \\
& GT & $1.30(0.55-3.05)$ & 0.653 \\
& T & $0.83(0.38-1.84)$ & 0.546 \\
\hline
\end{tabular}

$\mathrm{P}$-values were calculated by multinomial logistic regression. $\mathrm{BMl}$, body mass index.

exists between lower adiponectin levels and PCOS. Although this association was documented in several populations of various genetic backgrounds, this was the first study to investigate whether such an association exists in a Jordanian population. Furthermore, in addition to the association between PCOS and lower serum adiponectin protein levels, we found that a SNP (rs1501299) of the ADIPOQ gene was associated with PCOS. Specifically, in the multivariate analysis, we observed that the G allele of rs1501299 significantly increased the risk of PCOS in our population. This result supports our observation that lower adiponectin levels are associated with PCOS because previous reports have found that the $\mathrm{T}$ allele of rs1501299, presumably the protective allele, is associated with higher adiponectin expression levels. ${ }^{21)}$ The exact mechanism by which this allele affects adiponectin expression and/or PCOS risk remains undetermined. However, rs1501299 is an intronic variant located in an area of the intron that expresses long non-coding RNA. ${ }^{22)}$ These RNA species were shown to regulate gene expression ${ }^{23)}$ and potentially affect the expression of gene products, including adiponectin, that are mechanistically involved in PCOS development. This hypothesis, however, requires further testing in cellular and animal models.

Noteworthy was the finding that rs1501299 was the only SNP associated with PCOS among the three investigated SNPs. There could be several reasons for this: (1) rs1501299 may not be in linkage disequilibrium with the two other SNPs and thus could be part of a different chromosomal region that influences PCOS development; (2) neighboring population-specific SNPs could be present that could specifi- 
cally modify the effect of rs1501299 on PCOS but not those of the other two SNPs; and (3) the effect of the two other SNPs on PCOS could be smaller, so our population may not be large enough to detect an association.

The association of rs1501299 of the ADIPOQ gene with PCOS suggests that adiponectin levels and PCOS risk accordingly could be genetically determined. This observation supports those of the casecontrol study published by Mirza et al., ${ }^{24)}$ which found that females with a family history of PCOS are more likely to have lower adiponectin levels than women with no family history of PCOS. Both of our studies support the notion that adiponectin levels are genetically determined, which could also explain the genetic predisposition observed in several diseases where insulin resistance, influenced by adiponectin levels, plays an important role in disease etiology.

Panidis et al. ${ }^{25)}$ compared the adiponectin levels of women with PCOS to those of normally ovulating women without hyperandrogenism. In his analysis, he further stratified PCOS women into two groups by BMI ( $<25.0$ or $\left.\geq 25.0 \mathrm{~kg} / \mathrm{m}^{2}\right)$. In contrast to our results, Panidis et al. ${ }^{25}$ ) found that adiponectin levels were only lower in obese PCOS women relative to normally ovulating women or non-obese PCOS women and concluded that adiponectin might not contribute to the pathophysiology of PCOS. Although this study also examined the relationship between adiponectin and other metabolic markers of insulin resistance, the normal ovulating women group was relatively small $(n=15)$ and not matched to the PCOS women.

Our finding that adiponectin concentrations are lower in PCOS women relative to normally menstruating women in Jordan highlights approaches that can be used to mitigate the metabolic effects and/or clinical manifestations of this disease. Indeed, our results support the notion that PCOS and/or its complications could be treated by a pharmacological increase in serum adiponectin levels. Certain drug classes such as PPAR-gamma agonists can increase serum adiponectin levels and mitigate insulin resistance. ${ }^{26)}$ Indeed, thiazolidinediones (PPARgamma agonists) reportedly enhance the fertility of PCOS women. ${ }^{27}$ Considering the findings of this study, whether thiazolidinediones are superior drugs for treating fertility and other complications of PCOS than other insulin sensitizers (e.g., metformin) remains to be determined in controlled clinical trials.

Lower adiponectin levels are associated with obesity, ${ }^{13)}$ a major risk factor of PCOS development. ${ }^{2)}$ The fact that normally menstruating women recruited to participate in this study were matched with PCOS women by BMI excludes BMI as a confounding variable in our analyses. Body fat distribution is an important confounding variable of the relationship between adiponectin and PCOS. ${ }^{28)}$ Unfortunately, our study failed to include any indicators of body fat distribution such as waist-hip and visceral fat ratios. This, however, is a future direction of our research team.

In conclusion, our research group was the first to report lower levels of adiponectin in PCOS women in Jordan and the first to report that SNPs in the ADIPOQ gene modify PCOS risk. These results require further validation in larger studies across multiple institutions.

\section{CONFLICT OF INTEREST}

No potential conflict of interest relevant to this article was reported.

\section{ACKNOWLEDGMENTS}

Funding for this grant was provided through grant number (222/2016) awarded from the Deanship of Research at Jordan University of Science and Technology.

\section{REFERENCES}

1. Rotterdam ESHRE/ASRM-Sponsored PCOS Consensus Workshop Group. Revised 2003 consensus on diagnostic criteria and long-term health risks related to polycystic ovary syndrome. Fertil Steril 2004;81:19-25.

2. Bjorntorp P. The android woman: a risky condition. J Intern Med 1996; 239:105-10.

3. Ehrmann DA, Barnes RB, Rosenfield RL, Cavaghan MK, Imperial J. Prevalence of impaired glucose tolerance and diabetes in women with polycystic ovary syndrome. Diabetes Care 1999;22:141-6.

4. Sam S. Obesity and polycystic ovary syndrome. Obes Manag 2007;3: 69-73.

5. Escobar-Morreale HF, Botella-Carretero JI, Alvarez-Blasco F, Sancho J, San Millan JL. The polycystic ovary syndrome associated with morbid obesity may resolve after weight loss induced by bariatric surgery. J Clin Endocrinol Metab 2005;90:6364-9.

6. Hardy OT, Czech MP, Corvera S. What causes the insulin resistance underlying obesity? Curr Opin Endocrinol Diabetes Obes 2012;19:817.

7. Patel P, Abate N. Body fat distribution and insulin resistance. Nutrients 2013;5:2019-27.

8. Hotamisligil GS, Arner P, Caro JF, Atkinson RL, Spiegelman BM. Increased adipose tissue expression of tumor necrosis factor-alpha in human obesity and insulin resistance. J Clin Invest 1995;95:2409-15.

9. Dusserre E, Moulin P, Vidal H. Differences in mRNA expression of the proteins secreted by the adipocytes in human subcutaneous and visceral adipose tissues. Biochim Biophys Acta 2000;1500:88-96.

10. Cao H. Adipocytokines in obesity and metabolic disease. J Endocrinol 2014;220:T47-59.

11. Sun Y, Xun K, Wang C, Zhao H, Bi H, Chen X, et al. Adiponectin, an unlocking adipocytokine. Cardiovasc Ther 2009;27:59-75.

12. Diez JJ, Iglesias $P$. The role of the novel adipocyte-derived protein adiponectin in human disease: an update. Mini Rev Med Chem 2010;10: 856-69.

13. Kawano J, Arora R. The role of adiponectin in obesity, diabetes, and cardiovascular disease. J Cardiometab Syndr 2009;4:44-9.

14. Sheng T, Yang K. Adiponectin and its association with insulin resistance and type 2 diabetes. J Genet Genomics 2008;35:321-6.

15. Kondo H, Shimomura I, Matsukawa Y, Kumada M, Takahashi M, Matsuda M, et al. Association of adiponectin mutation with type 2 diabetes: a candidate gene for the insulin resistance syndrome. Diabetes 2002;51:2325-8.

16. Groth SW. Adiponectin and polycystic ovary syndrome. Biol Res Nurs 
2010;12:62-72.

17. Hivert MF, Manning AK, McAteer JB, Florez JC, Dupuis J, Fox CS, et al. Common variants in the adiponectin gene (ADIPOQ) associated with plasma adiponectin levels, type 2 diabetes, and diabetes-related quantitative traits: the Framingham Offspring Study. Diabetes 2008;57: 3353-9.

18. Yang WS, Yang YC, Chen CL, Wu IL, Lu JY, Lu FH, et al. Adiponectin SNP276 is associated with obesity, the metabolic syndrome, and diabetes in the elderly. Am J Clin Nutr 2007;86:509-13.

19. Biswas D, Vettriselvi V, Choudhury J, Jothimalar R. Adiponectin gene polymorphism and its association with type 2 diabetes mellitus. Indian J Clin Biochem 2011;26:172-7.

20. Verduci E, Scaglioni S, Agostoni C, Radaelli G, Biondi M, Manso AS, et al. The relationship of insulin resistance with SNP 276G $>$ T at adiponectin gene and plasma long-chain polyunsaturated fatty acids in obese children. Pediatr Res 2009;66:346-9.

21. Ramya K, Ayyappa KA, Ghosh S, Mohan V, Radha V. Genetic association of ADIPOQ gene variants with type 2 diabetes, obesity and serum adiponectin levels in south Indian population. Gene 2013;532:253-62.

22. Aken BL, Ayling S, Barrell D, Clarke L, Curwen V, Fairley S, et al. The Ensembl gene annotation system. Database (Oxford) 2016;2016: baw093.

23. Rinn JL, Chang HY. Genome regulation by long noncoding RNAs. Annu Rev Biochem 2012;81:145-66.

24. Mirza SS, Shafique K, Shaikh AR, Khan NA, Anwar Qureshi M. Association between circulating adiponectin levels and polycystic ovarian syndrome. J Ovarian Res 2014;7:18.

25. Panidis D, Kourtis A, Farmakiotis D, Mouslech T, Rousso D, Koliakos G. Serum adiponectin levels in women with polycystic ovary syndrome. Hum Reprod 2003;18:1790-6.

26. Sharabi Y, Oron-Herman M, Kamari Y, Avni I, Peleg E, Shabtay Z, et al. Effect of PPAR-gamma agonist on adiponectin levels in the metabolic syndrome: lessons from the high fructose fed rat model. Am J Hypertens 2007;20:206-10.

27. Froment $\mathrm{P}$, Touraine $\mathrm{P}$. Thiazolidinediones and fertility in polycystic ovary syndrome (PCOS). PPAR Res 2006;2006:73986.

28. Goropashnaya AV, Herron J, Sexton M, Havel PJ, Stanhope KL, Plaetke $\mathrm{R}$, et al. Relationships between plasma adiponectin and body fat distribution, insulin sensitivity, and plasma lipoproteins in Alaskan Yup'ik Eskimos: the Center for Alaska Native Health Research study. Metabolism 2009;58:22-9. 